\title{
LINGUISTICA CONTRASTIVA E TRADUZIONE LETTERARIA. ITALIANO E SPAGNOLO ${ }^{1}$
}

\author{
Manuel Carrera Díaz \\ Università di Siviglia
}

\begin{abstract}
Resumen: La lingüística contrastiva puede ser útil para la traducción literaria, puesto que ayuda al traductor a identificar y analizar correctamente los problemas de equivalencia entre dos o más lenguas. Siendo la lengua literaria un instrumento expresivo que explota al máximo todos los recursos lingüísticos, siempre será útil para el traductor el conocimiento de una base teórica que identifica los contrastes entre dos lenguas en todos los niveles relevantes para la interpretación del texto escrito literario: fonosimbólico, morfosintáctico, léxico, semántico, pragmático y gráfico.
\end{abstract}

Palabras clave: Lingǘstica contrastiva, traducción literaria, lengua italiana, lengua española.

Abstract: Contrastive linguistics can be useful for literary translation, since it helps the translator to correctly identify and analyze the problems of equivalence between two or more languages. Literary language is an expressive instrument that fully exploits all the linguistic resources. Theoretical knowledge about similarities and differences between languages at all levels (phonosymbolic, morphosyntactic, lexical, semantic, pragmatic and graphic level) can be therefore of great help to the translator.

Keywords: Contrastive linguistics, literary translation, italian language, spanish language.

\section{INTRODUZIONE}

La linguistica contrastiva, come ben sappiamo, studia le somiglianze e le differenze che intercorrono tra due lingue a tutti i livelli in cui queste sono paragonabili: fonetico, morfosintattico, lessicale, pragmatico, grafico. Questo tipo di approccio linguistico si è costituito, dopo alterne vicende, in un utile strumento di analisi nell'ambito della glottodidattica. Se sappiamo che gli usi

${ }^{1}$ Una prima versione di questo lavoro fu pubblicata in AAVV, L'insegnamento dell'italiano in Spagna: temi e prospettive (Convegno di Italianistica 20 gennaio 2011), Madrid, Istituto Italiano di Cultura, 2011, pp. 75-89. 
dell'imperfetto indicativo sono molto simili in italiano e in spagnolo, è superfluo stare a tormentare gli studenti con batterie di esercizi sull'uso di questo tempo; basta confermare, nello studiarlo, quel poco che è diverso tra le due lingue e quel molto che è simile per dedicare il resto del tempo ad argomenti che richiedono veramente un maggiore sforzo di analisi, comprensione ed esercitazione.

La linguistica contrastiva, dunque, viene in genere concepita come uno strumento di primo soccorso nella programmazione didattica dell'apprendimento linguistico, per renderlo più efficace ed economico. È chiaro che non è lo stesso insegnare lo spagnolo ad uno studente universitario italiano che ad una badante ucraina appena sbarcata in Italia. Dunque, una volta individuate le simmetrie e le dissimmetrie tra le due lingue, il docente è in grado di programmare adeguatamente le sue spiegazioni e i suoi sforzi didattici, almeno su un piano teorico.

Ma ci si può domandare se, oltre ad agire a questo livello elementare, la linguistica contrastiva non possa offrire degli spunti utili per portare a termine operazioni ben più complesse del semplice apprendimento di una lingua. E sto puntando in alto: può la linguistica contrastiva essere utile nell'ambito della traduzione letteraria? Può essere di aiuto nella formazione dei neotraduttori che seguono i frequentati e quotati corsi di Traduzione e Interpretariato che si tengono nelle università spagnole e italiane? Può, inoltre, essere di aiuto per un ormai affermato traduttore professionista che traduce in spagnolo testi di Petrarca o Moravia o che fa la versione italiana del Don Quijote?

A mio parere, la risposta alle tre domande precedenti è affermativa. Si è soliti distinguere e separare nettamente la traduzione strumentale dalla traduzione letteraria. Tradurre in spagnolo il manuale d'uso di una caffettiera Bialetti, il Codice della Strada o un articolo di medicina pubblicato su una rivista italiana è fare traduzione strumentale. Tradurre Il Principe di Machiavelli o i poemi di Montale è fare traduzione letteraria. La traduzione letteraria non è necessariamente più difficile della traduzione strumentale. Tradurre il manuale d'uso di una Fiat Punto richiede da parte del traduttore una conoscenza approfondita del lessico meccanico; tradurre il contenuto di una cartella clinica è un'operazione che può solo fare chi ha una conoscenza dettagliata del lessico medico, in genere, cioè, un medico che fa anche il traduttore; e così via per il resto del materiale testuale appartenente a linguaggi settoriali nei quali l'elemento fondamentale è la precisione e l'univocità linguistica. Apparentemente può sembrare un lavoro molto complesso, ma in realtà la sua riuscita si basa praticamente solo -anche se non è poco, comunque- sulla conoscenza del concreto sistema lessicale di quel settore; nel manuale d'uso di un computer, nel contratto di compravendita di una casa, in un testo legale o in un dossier clinico è piuttosto improbabile che compaia una onomatopea, una parolaccia o un'espressione ironica a doppio senso. Anzi, per definizione ciò è impossibile. 
Cosa accade, invece, ad un traduttore letterario? In genere, non ha a che fare con un lessico specialistico come quello precedentemente citato; cioè, non necessariamente deve avere una conoscenza esaustiva di un determinato settore lessicale. Ma ha comunque un problema, costituito dal fatto che la lingua letteraria è uno strumento che sfrutta al massimo le capacità espressive dello strumento linguistico. Questa è, probabilmente, la caratteristica più notevole della lingua letteraria: la capacità di sfruttare al massimo tutte le risorse linguistiche, il che significa che il traduttore letterario, pur non dovendo necessariamente essere un profondo esperto di un concreto settore linguistico, può trovarsi di fronte a non poche sorprese. Teoricamente non è uno specialista di niente, ma praticamente è costretto ad essere di volta in volta specialista nelle materie più inaspettate. Se la lingua è una Ferrari, noi parlanti comuni la guidiamo nella nostra vita quotidiana a 80 all'ora, mentre un letterato la può far sfrecciare a 300 all'ora, sfruttando al massimo la potenza del motore; il traduttore è tenuto così a seguire il percorso dell'autore alla stessa velocità di guida, ed è evidente che non si tratta di un'impresa facile.

Quali sono i livelli linguistici in cui, secondo la linguistica contrastiva, potrebbero emergere dei problemi per un traduttore letterario? Tanto per cominciare, almeno due sono da escludere: quello fonetico e quello gestuale, che nella lingua parlata sono così importanti. Ma un traduttore letterario ha a che fare solo con testi scritti; dunque, i problemi fonetici non lo riguardano, e quelli gestuali solo parzialmente, quando per caso si troverà a dover chiarire un gesto occasionalmente descritto nel testo.

\section{I LIVELLI DI ATTACCO}

\subsection{Il livello fonosimbolico}

Ma ciò è solo un piccolo vantaggio per il traduttore, perché i problemi si possono riscontrare già ad un livello pregrammaticale. Mi riferisco al piano fonosimbolico della lingua, cioè alle onomatopee e in parte alle interiezioni. Un traduttore scientifico o tecnico non ha questo problema: è impensabile che in un testo giuridico, in una cartella clinica o in un libro di fisica nucleare compaia un ahimè, un crunch! o un bau, bau! Ma un traduttore letterario maneggia testi in cui possono comparire svariate onomatopee e interiezioni. E non mi riferisco solo alla traduzione dei fumetti (che è sempre traduzione letteraria, anche nelle versioni testuali infantili, come Paperino o Topolino), regno incontrastato dell'onomatopea, ma anche ai testi letterari raffinati e appartenenti alle più alte manifestazioni letterarie. In un romanzo, le onomatopee possono comparire sia nelle parti dialogate, quando si riproduce una conversazione, sia nelle parti descrittive, quando si vuole raffigurare graficamente un determinato suono. Ma compaiono anche nell'ambito poetico, sia in maniera moderata (come i frequenti 
$\lceil O h !$ e $; A h !$ di Fray Luis de León) sia in forma cumulativa in testi in cui si cerca la ricreazione di certi effetti sonori (come ne La fontana malata di Palazzeschi), per non parlare di evidenti casi di sfruttamento delle onomatopee come quelli di Pascoli o Marinetti.

Ora, la linguistica contrastiva ci avverte che ogni lingua lessicalizza diversamente le onomatopee per lo stesso referente ${ }^{2}$. Il suono di uno starnuto umano è lo stesso ovunque, ma in italiano si sente e si rappresenta con eccì. mentre lo spagnolo lo fa con ¡achis! o jatchis! I galli fanno quiquiriquí in spagnolo e chicchiricchì in italiano, ma cocoricò in francese. Il suono del campanello è riiiing riiiing in spagnolo e driiin driiin in italiano. Ciò significa che il traduttore letterario deve prestare una particolare attenzione a non farsi trascinare dalle forme onomatopeiche del testo di partenza, perché sono sempre in agguato due possibili rischi:

a) Che la rappresentazione lessicale non coincida nelle due lingue, come abbiamo visto nei casi precedenti.

b) Che una lingua abbia assunto la rappresentazione lessicale da un'altra lingua (spesso, oggi, dall'inglese), e ciò potrebbe finire per integrare nella traduzione un prestito di secondo grado. La rappresentazione grafica del bacio sonoro in italiano è spesso Smack!, forma presa direttamente dall'inglese, mentre in spagnolo usiamo il più domestico ¡Mua!

Non è infrequente leggere traduzioni in cui le onomatopee e interiezioni risultano stridenti, appunto perché il traduttore non ha tenuto conto di questo fatto. Ma sembra essere una tentazione anche per i non traduttori. Un articolo pubblicato sul giornale $E l$ Pais $^{3}$ pochi anni fa recava questo titolo: ; Ups!, mi madre está en Facebook. Ma quando mai si è detto in spagnolo ;Ups!, direttamente derivato dall'inglese Oops!? Mai. Abbiamo detto sempre, in questo caso, ¡Huy!, ;Anda!, ecc.

Per la verità, in questo settore, il traduttore è abbandonato a sé stesso. Non esiste, purtroppo, una tabella di equivalenze delle onomatopee tra l'italiano e

2 “La convenzionalità dell'adattamento fonologico è un dato che può destare stupore: per il parlante nativo di una qualsiasi lingua le onomatopee appaiono talmente "naturali" che risulta difficile pensare che esse varino a seconda delle lingue. In realtà, in ogni segno linguistico, il rapporto tra significante (la forma, fonica o grafica) e significato (l'immagine mentale, il concetto ad essa associato) è arbitrario. In altri termini, sentendo pronunciare o leggendo una parola non se ne può dedurre il significato, a meno di non conoscerlo già. Le onomatopee non sono altro che dei casi in cui tale rapporto è "meno arbitrario" che in altri, nel senso che, per quanto più trasparenti, sono comunque legate alle specifiche culture in cui sorgono - come dimostrano le variazioni tra le diverse lingue." (V. Gheno, L'onomatopea, Accademia della Crusca. http:// www.accademiadellacrusca.it/it/lingua-italiana/consulenza-linguistica/domande-risposte/ lonomatopea ) 25-09-14.

3 In www.elpais.com, 12-01-2011. 
lo spagnolo, e questo, invece, sarebbe uno strumento validissimo per risolvere i dubbi in merito ${ }^{4}$. Se ne ha un qualche abbozzo, come l'utile accenno nella compilazione di Derek Abbott (Animal Sounds) consultabile su internet ${ }^{5}$, riferita ai versi degli animali e agli ordini per governarli, ma ciò, nell'ambito generale delle onomatopee, è ancora troppo poco. E quel che è stato appena detto per le onomatopee va anche applicato alle interiezioni, ostiche da tradurre e alle volte complicatissime da capire per uno straniero (si pensi, per esempio, agli svariati sensi di certe interiezioni spagnole derivate da forme verbali come ;Venga!, ¡Vaya!, ecc.). Ma la linguistica contrastiva ci aiuta, almeno, ad essere consapevoli di questa difficoltà.

\subsection{Il livello morfosintattico}

Un traduttore, ovviamente, è tenuto ad avere unottima conoscenza sia della lingua di partenza che di quella di arrivo. $\grave{E}$ la condizione minima, certamente. $\grave{E}$ chiaro, dunque, che deve conoscere ad un alto livello le strutture morfosintattiche delle due lingue, il che, teoricamente, sembrerebbe garanzia sufficiente di successo nel trasportare i contenuti da una lingua all'altra in maniera precisa ed efficiente.

Ma tradurre non è solo maneggiare in maniera asettica due lingue. Nel farlo, occorre tenere in considerazione due culture e due mondi ${ }^{6}$, il che spesso rende complessa una questione grammaticale semplice. Pensiamo, per esempio, a una questione elementare come il genere dei nomi e l'uso degli articoli corrispondenti. Sappiamo che in molti casi il genere dei nomi coincide, ed è dunque facilissimo tradurre il libro come el libro e la camicia come la camisa. E sappiamo anche che in molti altri casi il genere delle parole non è coincidente, di modo che il sangue sarà la sangre e il sale sarà la sal.

Ora, proviamo a tradurre il titolo di un articolo pubblicato pochi anni fa su La Repubblica: "Rogo nella villa del giudice Forleo"7. La prima tentazione del traduttore spagnolo sarebbe renderla come "Incendio en el chalet del juez Forleo", e la cosa può essere accettata finché non troviamo una foto del giudice Forleo. Vediamo, allora, che si tratta di Clementina Forleo, un giudice donna. La traduzione iniziale, dunque, non è corretta in spagnolo, perché c'è un'evidente contraddizione tra il genere dell'articolo e il sesso della persona indicata dal sostantivo, e pertanto va modificata come "Incendio en el chalet de la juez(a) Forleo".

${ }^{4}$ Per lo spagnolo vedi L. Gasca - R. Gubern, Diccionario de onomatopeyas del cómic, Madrid, Cátedra, 2008

${ }^{5}$ D. Abbott, Animal Sounds. Derek Abbott Home Page.http://www.eleceng.adelaide.edu.au/ Personal/dabbott/animal.html. 25-09- 14.

${ }^{6}$ M. Tricás Preckler, "Lingüística contrastiva y traducción. Aproximaciones interculturales", Synergies Espagne, 3(2010), p. 15.

7 In www.repubblica.it, 5-01-2011. 
Perché è avvenuta questa iniziale confusione? Ciò è spiegabile in quanto il traduttore non ha tenuto conto di una constatazione della linguistica contrastiva dello spagnolo e dell'italiano secondo la quale, tendenzialmente, il genere nella lingua italiana è più grammaticale, mentre in spagnolo risulta più personale ${ }^{8}$. Cioè, in spagnolo, gli indicatori grammaticali del genere tendono ad avvicinarsi di più al sesso reale della persona, e ciò è evidente nel caso dei nomi che indicano mestieri tradizionalmente maschili attualmente esercitati anche dalle donne. Dunque, "il Rettore Luisa Bianchi” sarà "la Rectora Luisa Bianchi”. E bisogna prestare particolare attenzione quando non compare un nome di battesimo (come succedeva nel caso del giudice Forleo) o quando questo per noi è opaco: un costrutto come "il ministro dei Trasporti islandese Asdís Morkelsdottir" va benissimo in italiano, che grammaticalmente non tiene conto del sesso reale del suddetto ministro, ma non funziona in spagnolo perché il tale ministro è una donna, come palesa il suo cognome terminato in -dottir ("la ministra de Transportes islandesa, Asdís Morkelsdottir"). Non basta la grammatica, dunque, per il traduttore: ci vuole anche la linguistica contrastiva e la conoscenza del mondo.

E non è questo l'unico caso in cui l'uso dell'articolo e la relativa traduzione non può essere ridotta ad una questione strettamente grammaticale. Sappiamo, per esempio, che l'articolo determinativo è meno determinativo in italiano che in spagnolo, per cui una frase come "Vanno al ristorante due volte la settimana" non va tradotta come "Van al restaurante...", bensì come "Van a un restaurante..."; sappiamo anche che le norme che regolano la comparsa dell'articolo davanti a nomi e cognomi o a sostantivi in serie enumerative sono diverse in italiano e in spagnolo, ecc. Fattori questi, se mi si permette di insistere, previsti dalla grammatica contrastiva e che non dovrebbero indurre in confusione un traduttore ferrato.

\subsection{Il livello lessicale}

Enormi difficoltà di traduzione si possono incontrare anche a livello lessicale. Innanzitutto, con il noto problema dei falsi amici, evidente anche per uno studente di primo livello e persino elemento di curiosità aneddotica per coloro che di lingue non se ne intendono. L'italiano e lo spagnolo presentano un alto indice di coincidenze lessicali, ma come sappiamo, il collegamento formasignificato non sempre è equivalente nelle due lingue.

È chiaro che un traduttore italiano ben preparato sa che un dormitorio spagnolo può essere sia un luogo di riposo collettivo dove dormono parecchie persone (in un collegio o caserma, per esempio) sia una camera da letto e anche l'insieme dei mobili all'interno di una casa o appartamento. Se vengono ignorate queste cose,

8 Sulla lapide della tomba di Oriana Fallaci al Cimitero Evangelico agli Allori di Firenze c’è scritto, semplicemente, oltre alle date, "ORIANA FALLACI. SCRITTORE”. 
non si è traduttori, bensì semplici dilettanti poco preparati. Ma un traduttore letterario spesso si trova a doversi confrontare con testi di altri periodi e deve quindi tenere presenti le caratteristiche della lingua in quel concreto momento storico. Storicamente, per esempio, l'italiano è stato, per ragioni ovvie, molto più aperto, fin dalle origini, all'influsso del latino, e dunque all'adattamento di parole latine al lessico romanzo ${ }^{9}$. Non sono infrequenti, dunque, i latinismi semantici, che richiedono una particolare attenzione da parte del traduttore. Dunque, quando Petrarca nel sonetto 265 parla de "la superna strada", non possiamo tradurlo come "el supremo camino", perché "supremo", in spagnolo, ha acquisito un'altra valenza semantica (Tribunal Supremo, turrón supremo de Jijona), bensí come "el camino más alto, el de más arriba". E quando Sannazzaro, nell'Arcadia, si riferisce alle "lascive pecorelle", non vuole censurare affatto dal punto di vista morale queste bestiole, perché non fa altro che usare un latinismo semantico: non "las lascivas ovejitas" in spagnolo, bensì "las juguetonas ovejitas".

Le modifiche morfologiche che si operano sulle parole possono creare anche notevoli problemi al traduttore se questo non è avvertito sulle differenze contrastive che intercorrono tra le due lingue. Sappiamo, per esempio, che il superlativo in -issimo è in italiano più frequente e più ampiamente usato che non in spagnolo, oltre al fatto che in spagnolo non si ama ripeterlo in maniera ravvicinata né negarlo: se sono stanchissimo posso dire in spagnolo che "estoy cansadísimo", ma una "bellissima e giovanissima ragazza" sarà in spagnolo, probabilmente, "una chica muy joven y guapa", e "una non chiarissima faccenda" sarà "un asunto no muy claro".

Lo stesso dicasi nel caso dei diminutivi. In generale, si può dire che in italiano il diminutivo mantiene abbastanza bene l'originale funzione di indicare una cosa più piccola: un telefonino, una radiolina, un motorino. In spagnolo, ormai, questa funzione si ha praticamente in forma solo lessicalizzata (plazuela, avioneta, patillas), perché in genere i diminutivi conferiscono più o meno forti connotazioni affettive alle parole che li subiscono. Il traduttore, soprattutto nella direzione italiano-spagnolo, deve essere consapevole di queste differenze per evitare che il suo testo terminale suggerisca sfumature che non sono affatto presenti nel testo originale.

Alcuni diminutivi spagnoli presentano notevoli difficoltà di traduzione in italiano (e in altre lingue). Se io dico "María estaba allí solita", posso tradurlo come "Maria era lì sola soletta". Ma altri casi simili in cui il suffisso diminutivo si aggiunge a un participio -operazione non permessa in italiano- sono più complessi. È la domanda che si faceva Jorge Luis Borges in una famosa conferenza del 1980: come tradurre in un'altra lingua l'espressione "Estaba sentadita", dove voglio indicare che era seduta e che io la vedo lì con affetto e tenerezza? Diversi participi aggettivali dello spagnolo ammettono il suffisso

9 Vedi M. Morreale, Homenaje a Fray Luis de León, Salamanca, Ediciones de la Universidad de Salamanca, 2007, p. 455. 
diminutivo (calladito, sentadito, agarradito, cargadito) perché i parlanti li sentono come aggettivi modificabili, mentre gli italiani vedono le forme omologhe come puramente verbali o come aggettivi non modificabili:

- Quietecito, ¿̨eh? = Fermo, capito?

- Tú estate calladito $=\mathrm{Tu}$ te ne stai bello zitto

- Este año mis padres han venido cargaditos de regalos = Quest'anno i miei genitori sono venuti belli carichi di regali

L'interpretazione, poi, di questi diminutivi, richiede, nell'ambito della traduzione, una certa finezza interpretativa, perché possono avere svariati significati: intensivo (cargadito), affettivo (sentadita) o chiaramente intimidatorio (calladito, quietecito $)^{10}$.

Anche il trattamento dei forestierismi può essere diverso nelle due lingue. In un testo scritto attuale è molto probabile che ci siano più anglicismi in italiano che non in spagnolo. Il traduttore, dunque, dovrà tener presente la statistica media di tolleranza di ciascuna lingua rispetto ai termini presi di peso da un'altra lingua. Ed è anche probabile che le sigle derivate da termini stranieri siano diverse (AIDS - SIDA).

\subsection{Il livello semantico-culturale}

A volte, certe parole o gruppi di parole assumono in una lingua cariche semantiche che non hanno affatto corrispondenza con le omologhe parole di un'altra lingua. In questi casi una traduzione letterale è fuorviante perché nel testo terminale le parole non avrebbero la carica semantica e referenziale dei termini originali.

Pensiamo, per esempio, agli aggettivi relativi ai colori, che possono acquisire una simbologia diversa a seconda delle culture e delle lingue. Questi aggettivi indicano, sì, una modalità cromatica, ma possono anche essere abbinati a significati molto specifici di una cultura ${ }^{11}$. Se, per esempio, una persona subisce l'effetto di un colpo su una parte del corpo e questa prende una tonalità scura, in italiano la si vede con un tono cromatico vicino al blu, mentre in spagnolo la si percepisce vicina al viola:

10 "El adjetivo "agarraditos" no significa únicamente 'muy agarrados', sino que sugiere también 'de buen grado' o 'en actitud cariñosa'. Se aplican valores similares a muchos otros casos, y se obtienen así diversos matices en los que se transmiten —en contextos también distintosestima,benevolencia, simpatía, complacencia, complicidad, pero también ironía, desaire o censura en ciertas situaciones" (Real Academia Española, Nueva gramática de la lengua española, Madrid, Espasa Libros, 2009, vol. I, p. 656)

${ }^{11}$ Vedi, per quanto riguarda l'italiano e lo spagnolo, M. Galiñanes Gallén, "La traducción de los colores en italiano y en español”, RedELE, 4(junio de 2005). (http://www.mecd.gob.es/dctm/ redele/Material-RedEle/Revista/2005_04/2005_redELE_4_03Galinanes.pdf?documentId=090 1e72b80dfb085). 25.9.2014. 
Aveva un occhio blu = Tenía un ojo morado

Avere le labbra blu $=$ Tener los labios lividos o amoratados

$\mathrm{E}$ i riferimenti a un dato colore possono essere esclusivi di solo una delle due lingue: un auto blu è un coche oficial, e un colletto blu è, semplicemente, un obrero. Prendiamo il caso del bianco, che sembra essere un colore universalmente innocente: il bianco dell'uovo è la clara del huevo; un risultato in bianco è un empate a cero; i capelli bianchi sono las canas, e i colletti bianchi sono gli oficinistas. Allo stesso modo, il termine di paragone in riferimento a un colore può variare notevolmente da una lingua all'altra: rosso come un peperone in spagnolo va tradotto come rojo como un tomate.

Lo stesso dicasi, per fare un altro esempio, per la traduzione dei numerali, che non sembrerebbero suscettibili di cambiamento nel travaso da una lingua all'altra ${ }^{12}$. Se io dico che "María tiene 28 años" o che "De Madrid a Sevilla hay 538 kilómetros", è ovvio che nella traduzione italiana questi numeri vanno scrupolosamente rispettati ("María ha 28 anni", "Da Madrid a Siviglia ci sono 538 chilometri"). Qualche dubbio può insorgere, però, quando vediamo che un millepiedi è soltanto un ciempiés in spagnolo. $\mathrm{O}$, soprattutto, quando abbiamo a che fare con gli usi non-simbolici ${ }^{13}$, cioè approssimativi, dei numeri: "fare quattro passi" = "dar una vueltecita, un paseíto", "fare due chiacchiere" = "charlar un rato", "in un quattro e quattrotto" = "en un abrir y cerrar de ojos". La casistica, qui, è estremamente ampia: il numerale può essere presente in una lingua e assente nell'altra, come nei casi precedentemente citati e in tanti altri che si potrebbero aggiungere: la tredicesima è, in spagnolo, la paga extraordinaria, e una ventiquattrore è un maletín; il livello della quantificazione può cambiare in alto o in basso: "dirgliene quattro" = "cantarle las cuarenta"; la categoria del numerale (cardinale, ordinale, frazionario...) può essere diversa: "il primo marzo" = "el uno de marzo"; può variare l'unità di misura: "un etto di prosciutto cotto = "cien gramos de jamón de York"; "due etti e mezzo = un cuarto"; le procedure di indicazione dell'approssimazione possono essere diverse: "una settantina di ragazzi" = "unos setenta chicos"; specifiche procedure quantificative possono essere esclusive di una delle due lingue: "un tredicenne" = "un cbico de trece años"; ecc. Non si può non coincidere, dunque, con E. Strudsholm quando sostiene che "visto nel suo uso concreto, ogni singolo numero ha un significato su cui non si può discutere, ma (...) il significato di molti numerali dipende dal contesto in cui essi sono usati: in alcuni contesti i numerali espandono il significato 'canonico' in diverse direzioni, quantitativamente inferiori o superiori, ed è proprio qui che compaiono le sfide del traduttore" 14 .

12 Vedi C. Bazzanella, Numeri per parlare, Bari, Laterza, 2011.

13 Nella terminologia di Dehaene, citato da C. Bazzanella, cit., p. 7.

${ }^{14}$ E. Strudsholm, "Quanto è difficile tradurre i numerali: il caso del danese", in. C. Bazzane1la, cit. , p. 124. 
Si potrebbero moltiplicare gli esempi in riferimento ad altri campi semantici diversi da questi appena considerati, ma che condividono con essi queste divergenze semantico-culturali. $\mathrm{E}$ dunque una cosa è chiara: un traduttore deve stare sempre attento a non cadere in una stretta letteralità quando si ha a che fare con casi come questi.

\subsection{Il livello pragmatico comunicativo}

Dicevamo precedentemente che il traduttore letterario spesso ha a che fare con brani scritti che riproducono la lingua parlata, cioè, dialoghi e interazioni comunicative dirette, quando per esempio sta traducendo un romanzo o un testo teatrale. Deve perciò fare attenzione a certi tratti che si manifestano con particolare intensità nella lingua parlata, come nel caso dei segnali Oew Çdiscorsivi, cioè di quegli elementi linguistici che non hanno una chiara funzione sintattica e si limitano a guidare le inferenze linguistiche. Un caso caratteristico, tanto per fare un esempio, è quello della particella spagnola pues, che ha una straordinaria varietà di funzioni che vanno tradotte con precisione per evitare che il testo terminale risulti strano al lettore.

Le convenzioni comunicative-sociali di ciascuna lingua vanno anche tenute in considerazione nell'ambito della traduzione per mantenere il carattere naturale del testo originale, perché il non farlo potrebbe suggerire connotazioni fuorvianti. Immaginiamo questo breve dialogo italiano:

-Buongiorno, avvocato.

-Buongiorno, ingegnere, come sta?

-Bene, bene. Ah, ecco che arriva anche il nostro caro commendatore.

Una traduzione spagnola dovrebbe eliminare i riferimenti alle professioni, perché l'indicazione di queste salvo in casi molto speciali (profesor, doctor, ecc.) non ha rilevanza nell'interazione comunicativa degli ispanofoni, almeno in Spagna. Includere gli appellativi di mestiere potrebbe suggerire al lettore ispanofono che la conversazione punta su fattori professionali o che il lavoro di ciascuno degli attori ha particolare rilevanza, il che ovviamente non è così.

Devono inoltre essere tenute presenti le convenzioni socio-comunicative che regolano in ciascuna lingua le relazioni interpersonali in specifiche situazioni come potrebbero essere gli incontri e i congedi. Noi docenti spieghiamo ai nostri allievi che ciao è un'espressione di saluto bidirezionale, perché si usa sia per l'incontro che per il congedo, mentre buonanotte è soltanto un saluto monodirezionale da congedo. Ma spesso ci dimentichiamo di segnalare che buongiorno e buonasera sono, come ciao, bidirezionali, cioè si usano sia per il saluto che per il congedo, il che non è il caso dello spagnolo, che in genere usa buenos dias e buenas tardes solo per il saluto. Una traduzione spagnola dall'italiano 
in cui gli interlocutori dei dialoghi si congedano dicendo buenos días e buenas tardes verrà perfettamente capita e interpretata da un ispanofono, ma vi troverà, senza dubbio, qualcosa di strano e non naturale.

Di nuovo, per il traduttore, non è sufficiente il dominio delle due lingue: ci vuole anche la consapevolezza delle dissimmetrie, la conoscenza del mondo e l'interpretazione adeguata delle situazioni comunicative.

\subsection{Il livello grafico}

Il traduttore letterario lavora, come dicevamo, con testi scritti, e scritta è la resa finale del suo lavoro. Bisognerà tenere presenti, dunque, anche le convenzioni e gli usi grafici di ciascuna lingua. Mi riferisco, specificamente, ai segni di punteggiatura ${ }^{15}$. Sono praticamente gli stessi in italiano ed in spagnolo, con l'eccezione del punto esclamativo e del punto interrogativo iniziali dello spagnolo. Ma le procedure di interpunzione possono essere leggermente diverse nell'una e nell'altra lingua ${ }^{16}$. Varia, per esempio, l'uso della virgola; sembra più frequente in italiano l'uso dei due punti. Per indicare le diverse battute di un dialogo in italiano si possono usare le virgolette oppure la lineetta lunga, mentre in spagnolo è universale ed esclusivo l'uso della lineetta lunga.

Il traduttore, dunque, non dovrà legarsi in maniera ossessiva alla punteggiatura del testo originale, bensì operare le opportune modifiche per rendere fluido e naturale il suo testo terminale, e seguendo esclusivamente le convenzioni grafiche della lingua in cui questo è scritto.

Due lingue, due culture, due mondi referenziali, e tante diversità e dissimmetrie a tutti i livelli linguistici, anche tra lingue affini come l'italiano e lo spagnolo. In un lavoro complesso come quello della traduzione, tutte le risorse e possibilità di analisi differenziale vanno messe a frutto, e non ultima tra di esse la linguistica contrastiva, come si diceva all'inizio.

15 Sullo sviluppo storico dei segni di interpunzione in italiano e in spagnolo, vedi B. Mortara Garavelli (a cura di), Storia della punteggiatura in Europa, Bari, Laterza, 2008.

16 Vedi M. Carrera Díaz, Grammatica spagnola, Laterza, Bari, 2010, pp. 131-137. 
